\title{
Electroweakino and slepton pair production at the LHC in NLO+NLL with resummation-improved PDFs
}

\author{
Juri Fiaschi, Michael Klasen ${ }^{* \dagger}$ \\ Institut für Theoretische Physik, Westfälische Wilhelms-Universität Münster, \\ Wilhelm-Klemm-Straße 9, 48149 Münster, Germany \\ E-mail: fiaschi@uni-muenster.de, michael.klasenduni-muenster.de
}

\begin{abstract}
Using parton density functions (PDFs) with threshold-resummation improvement, we consistently calculate higgsino/gaugino and slepton pair production at next-to-leading order and nextto-leading logarithmic accuracy at the LHC. The smaller PDF uncertainty of the global PDF sets is exploited with a factorisation method, which also avoids complications arising in the refitting of threshold-resummation improved PDF replicas in Mellin space. We explicitly take into account the reduction of the scale uncertainty due to the resummation. We show that the resummation contributions in the PDF fits partially compensate the cross section enhancements induced by those in the partonic matrix elements.
\end{abstract}

European Physical Society Conference on High Energy Physics - EPS-HEP2019 -

10-17 July, 2019

Ghent, Belgium

\footnotetext{
* Speaker.

${ }^{\dagger}$ Work supported by the BMBF under contract 05H18PMCC1 and the DFG through the Research Training Group 2149 "Strong and weak interactions - from hadrons to dark matter".
} 


\section{Motivation}

Supersymmetry (SUSY) is an extension of the Standard Model (SM) that is not only well motivated at high scales as the largest possible extension of the Poincare symmetry, that includes supergravity and is an essential part of string theory, but also at the weak scale, where it proposes a solution to the hierarchy, unification and dark matter problems [1]. Recently, simplified models have been employed to not only focus on the relevant SUSY particle sectors, but also as prototypes for many more minimal Beyond-the-SM theories [2]. Therefore, the LHC experiments ATLAS and CMS actively search for SUSY particles in simplified scenarios and will continue to do so with higher luminosity in the upcoming runs.

To fully exploit the experimental discovery potential, precise theoretical calculations of SUSY particle production are required. They have been performed at fixed, next-to-leading order (NLO) and with resummation methods to all orders at next-to-leading-logarithmic (NLL) accuracy and beyond for sleptons [3, 4], gauginos/higgsinos [3, 5], squarks and gluinos [6, 7] and associated gluino-gaugino/higgsino production $[8,9]$. Note that the sensitivity for this last process at the High-Luminosity LHC has recently been studied [10]. The calculations for the electroweak and semi-strong channels have been made public with the code RESUMMINO [11]. They generally increase the total cross sections and reduce the scale dependence. In our previous work, the parton density function (PDF) uncertainty remained at NLO, since it was not reduced by the resummation in the hard matrix elements of the SUSY particle production processes.

\section{Formalism}

PDFs with resummation improvement are now available with the NNPDF3.0 set [12]. However, as NLO+NLL calculations could not be used for all processes employed in typical global PDF fits, the resummation-improved fit had to be performed with the reduced data set of deepinelastic scattering, Drell-Yan and top-pair production data. To consistently show the impact of the resummation contributions, an NLO analysis of the same data set has also been performed.

The NNPDF analyses employ a replica method that is known to be problematic in Mellin space, where PDFs are required at all values of $x$. In particular, outliers can produce unphysical (e.g. negative) cross sections. Also, due to the reduced data set the resummation-improved PDF analysis presented a larger, not smaller uncertainty than the global analysis.

To avoid both shortcomings, a method based on ratios of NLL and NLO cross sections with reduced and global PDF fits can be employed [13]. By defining a $K$-factor as

$$
K=\frac{\sigma(\mathrm{NLO}+\mathrm{NLL})_{\mathrm{NLO} \text { global }}}{\sigma(\mathrm{NLO})_{\mathrm{NLO} \text { global }}} \cdot \frac{\sigma(\mathrm{NLO}+\mathrm{NLL})_{\mathrm{NLO}+\mathrm{NLL} \text { reduced }}}{\sigma(\mathrm{NLO}+\mathrm{NLL})_{\mathrm{NLO} \text { reduced }}}
$$

(approximate) NLO+NLL cross sections with NLO+NLL PDFs can be obtained with

$$
\sigma(\mathrm{NLO}+\mathrm{NLL})_{\mathrm{NLL}+\mathrm{NLO} \text { global }}=K \cdot \sigma(\mathrm{NLO})_{\mathrm{NLO} \text { global }}
$$

This allows also to obtain (approximate) NLO+NLL global PDF errors by varying the NLO global PDFs in $\sigma(\mathrm{NLO})_{\mathrm{NLO}}$ global, and at the same time to eliminate replicas with unphysical behaviour. The renormalization and factorization scales are still varied with the seven-point method directly 
in $\sigma(\mathrm{NLO}+\mathrm{NLL})$. The total theoretical uncertainty is obtained by adding PDF and scale errors in quadrature. This method can not only be applied to total cross sections $\sigma$, but also to distributions in the invariant mass $M$ of the produced SUSY particle pair $d \sigma / d M_{\tilde{\ell} \tilde{\ell}, \tilde{\chi} \tilde{\chi}}$.

We apply this method to SUSY particle production in simplified scenarios of the Minimal Supersymmetric Standard Model (MSSM). For sleptons, we assume pure left-handed mass-degenerate selectrons and smuons and right-handed or maximally mixed staus. For neutralinos and charginos, we assume a natural SUSY spectrum with Higgsino masses below $1 \mathrm{TeV}$ and a compressed spectrum of $m_{\tilde{\chi}_{1}^{ \pm}} \simeq m_{\tilde{\chi}_{2}^{0}} \simeq m_{\tilde{\chi}_{1}^{0}}$ as well as heavier gauginos. The processes that are implemented in RESUMMINO include the electroweak processes $p p \rightarrow \tilde{\ell} \tilde{\ell}^{*}(\ell=e, \mu), \tilde{\tau}_{R} \tilde{\tau}_{R}^{*}, \tilde{\tau}_{1} \tilde{\tau}_{1}^{*} ; p p \rightarrow \tilde{\chi}_{i}^{ \pm} \tilde{\chi}_{j}^{\mp}$, $\tilde{\chi}_{i}^{ \pm} \tilde{\chi}_{j}^{0}, \tilde{\chi}_{i}^{0} \tilde{\chi}_{j}^{0}(i, j=1,2)$; the semi-strong processes $p p \rightarrow \tilde{g} \tilde{\chi}_{j}^{ \pm, 0}, \tilde{q}_{i} \tilde{\chi}_{j}^{ \pm, 0}$ (in progress); and the GUT processes $p p \rightarrow Z^{\prime} \rightarrow \ell \bar{\ell}, p p \rightarrow W^{\prime} \rightarrow \ell \bar{v}$. Since the produced sleptons, charginos and neutralinos decay as $\tilde{\ell} \rightarrow \ell \tilde{\chi}_{1}^{0}, \tilde{\chi}_{1}^{ \pm} \rightarrow W^{ \pm} \tilde{\chi}_{1}^{0} \rightarrow \ell \bar{v} \tilde{\chi}_{1}^{0}$ and $\tilde{\chi}_{2}^{0} \rightarrow Z \tilde{\chi}_{1}^{0} \rightarrow \ell \bar{\ell} \tilde{\chi}_{1}^{0}$, the experimental signatures involve (sometimes soft) leptons and (possibly moderate) missing transverse energy $E_{T}$. We do not include the corresponding branching ratios and efficiencies, since they are usually taken into account experimentally [14].

\section{Slepton/stau production}

For pairs of left-handed sleptons with $564 \mathrm{GeV}$ mass, we show the invariant distribution in Fig. 1 (left) in leading order (LO) (green dotted), NLO (blue dashed) and NLO+NLL (red full)
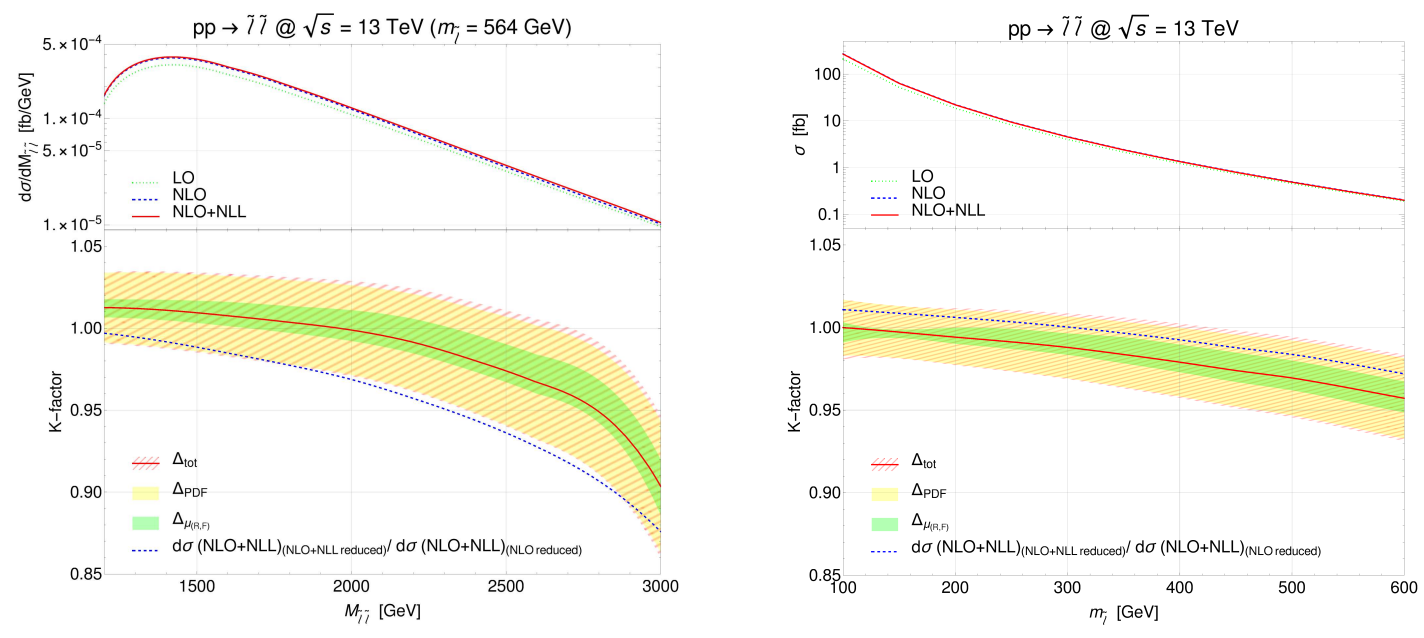

Figure 1: Left: Invariant-mass distributions of left-handed slepton pairs produced at the LHC with 13 $\mathrm{TeV}$ center-of-mass energy. Right: Corresponding total cross sections as a function of the slepton mass. Shown are results at LO (green dotted), NLO (blue dashed) and NLO+NLL (red full) together with the corresponding $K$-factors and their uncertainties (lower panels).

together with the corresponding $K$-factors and their uncertainties (lower panel). At the peak, the NLO corrections increase the LO prediction by $16 \%$, while the NLL contributions increase it in addition by $2-3 \%$. In the lower panel, the red line shows the full $K$-factor, while the blue dashed line indicates the effect of resummation in the PDFs only. As one can see, they partially compensate the resummation effects in the matrix elements. 
Fig. 1 (right) shows the corresponding total cross section as a function of the slepton mass. In this case, the NLO+NLL cross sections with NLO+NLL PDFs are smaller by $\sim 4 \%$. Assuming three generations of mass-degenerate sleptons, ATLAS can now exclude masses up to $700 \mathrm{GeV}$, while for left-handed selectrons and smuons individually the limits reach $550 \mathrm{GeV}$. The sensitivity drops sharply with the mass difference of sleptons and neutralinos [15].

The corresponding total cross sections for right-handed and maximally mixed stau pairs are shown in Fig. 2. The total cross sections for right-handed staus (left) are smaller than those for
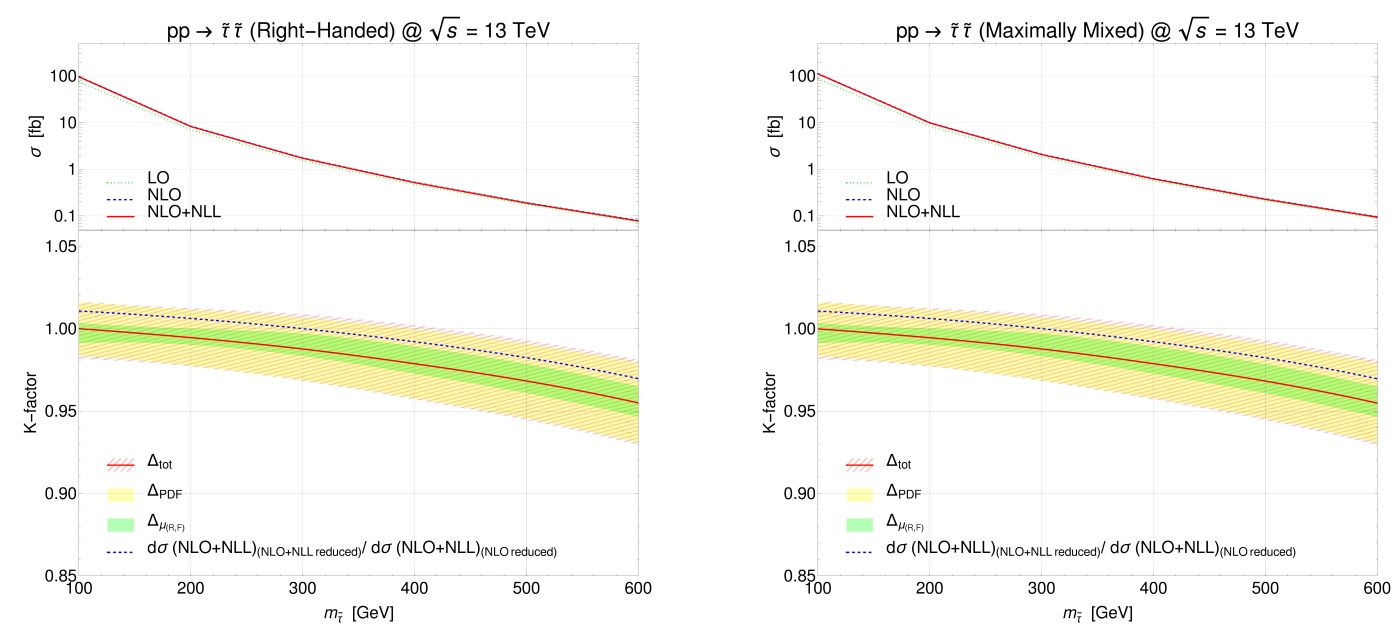

Figure 2: Left: Total cross sections of right-handed stau pairs produced at the LHC with $13 \mathrm{TeV}$ centerof-mass energy as a function of the stau mass. Right: Same for maximally mixed stau pairs. Shown are results at LO (green dotted), NLO (blue dashed) and NLO+NLL (red full) together with the corresponding $K$-factors and their uncertainties (lower panels).

left-handed staus by almost a factor of three, those for maximally mixed staus (right) by about a factor of two. The individual corrections and uncertainties from the higher-order predictions show a very similar behavior as in the left-handed case. Current CMS limits on stau masses, obtained with an integrated luminosity of $\mathscr{L}=77.2 \mathrm{fb}^{-1}$, lie at $m_{\tilde{\tau}}>90 \ldots 200 \mathrm{GeV}$ [16].

\section{Higgsino/gaugino production}

Turning to electroweakino production, we first show in Fig. 3 the invariant mass distribution for light higgsino pairs of $204 \mathrm{GeV}$ mass. They are obtained by setting the higgsino mass parameter $\mu$ to $200 \mathrm{GeV}$ while keeping the bino and wino mass paramters $M_{1,2}$ at $1 \mathrm{TeV}$. In this compressed scenario, the mass differences of second-lightest neutralino and lightest chargino amount to only $m_{\tilde{\chi}_{2}^{0}}-m_{\tilde{\chi}_{1}^{ \pm}} \approx m_{\tilde{\chi}_{1}^{ \pm}}-m_{\tilde{\chi}_{1}^{0}} \approx 5 \mathrm{GeV}$. At low invariant mass $M_{\tilde{\chi} \tilde{\chi}}$, the NLO corrections increase the LO prediction by $20 \%$, while the NLL contributions increase it in addition by $1-4 \%$.

The corresponding total cross sections are shown in Fig. 4 as a function of the higgsino mass for positive charginos (left) and negative charginos (right). At a $p p$ collider like the LHC, the former have of course the larger cross section, which is increased by using the NLO+NLL PDFs. Negative charginos not only have a smaller total cross section, but it is also reduced by using NLO+NLL PDFs. 


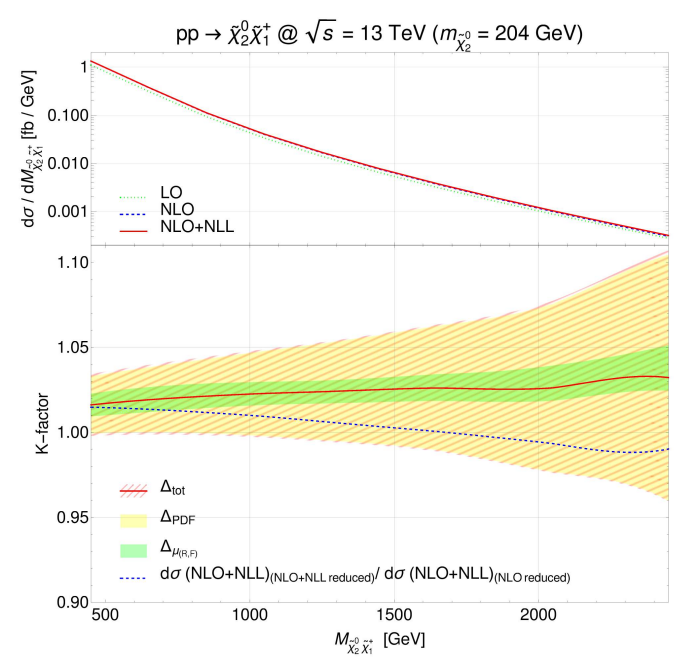

Figure 3: Invariant-mass distributions of light higgsino pairs produced at the LHC with $13 \mathrm{TeV}$ center-ofmass energy. Shown are results at LO (green dotted), NLO (blue dashed) and NLO+NLL (red full) together with the corresponding $K$-factors and their uncertainties (lower panels).
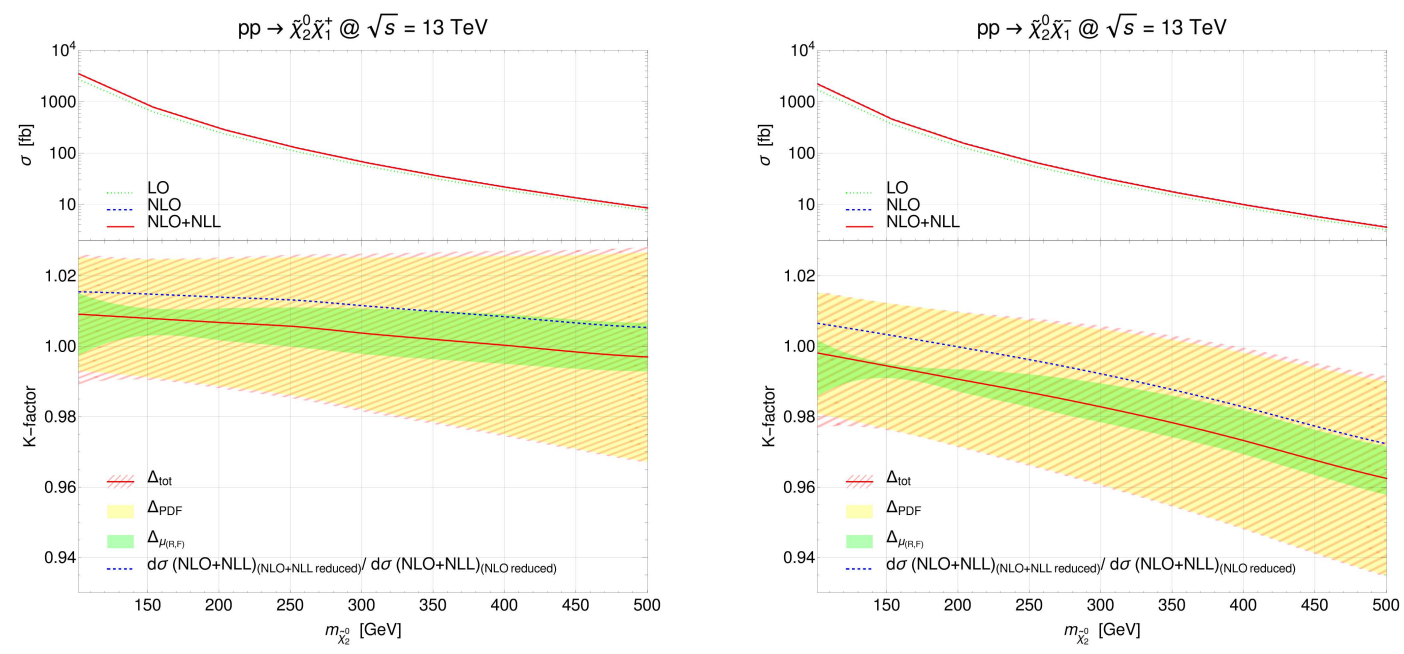

Figure 4: Left: Total cross sections of positive charginos and neutralinos produced at the LHC with $13 \mathrm{TeV}$ center-of-mass energy as a function of the higgsino mass. Right: Same for negative charginos. Shown are results at LO (green dotted), NLO (blue dashed) and NLO+NLL (red full) together with the corresponding $K$-factors and their uncertainties (lower panels).

Finally, we look at gauginos and their invariant mass distribution in Fig. 5 (left). Here, the NLO corrections increase the LO prediction by as much as $30-80 \%$, and the NLL contributions add another $6-10 \%$. The reason is that these winos are taken to be considerably heavier than the higgsinos, so that their production occurs closer to threshold, inducing larger logarithmic corrections. For this reason also the PDF uncertainties are larger in this case. The total gaugino cross sections are shown in Fig. 5 (right) as a function of the gaugino mass. Here we observe only a small effect from the resummation in the PDFs. 

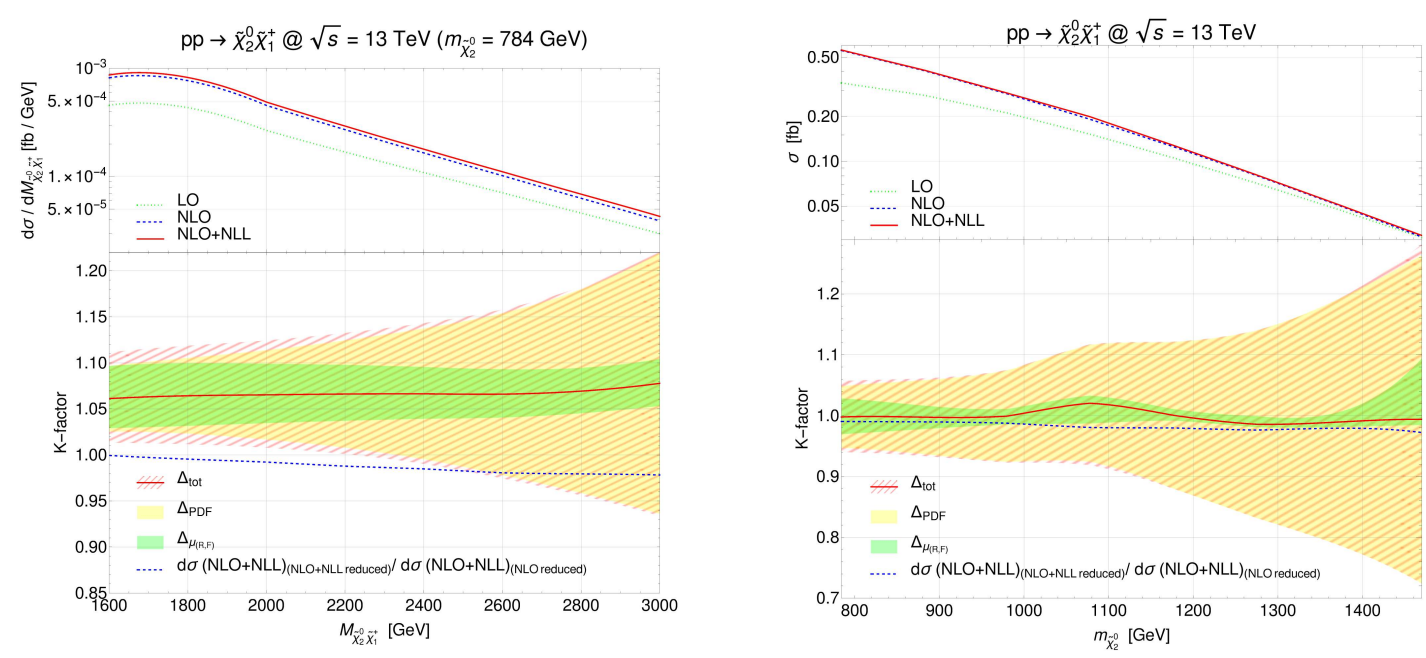

Figure 5: Left: Invariant-mass distributions of heavier gaugino pairs produced at the LHC with $13 \mathrm{TeV}$ center-of-mass energy. Right: Corresponding total cross sections as a function of the neutralino mass. Shown are results at LO (green dotted), NLO (blue dashed) and NLO+NLL (red full) together with the corresponding $K$-factors and their uncertainties (lower panels).

\section{Conclusion}

To summarize, the public code RESUMMINO now allows to produce predictions at NLO+NLL for electroweak SUSY particle production using also resummed PDFs. Although one would in principle expect this to lead to smaller PDF uncertainties, the fact that these PDFs had to be obtained with a reduced data set leads instead to larger PDF uncertainties. However, the advantages of resummation corrections in the PDFs and large data sets entering global PDF analyses can be combined by using a method based on cross section ratios, i.e. $K$-factors.

Large cross sections were predicted in particular for left-handed sleptons and light higgsinos, while they are naturally smaller for right-handed sleptons and heavier winos. For the latter, we found instead large resummation corrections close to threshold. In general, resummation corrections in the matrix elements were found to be partially compensated by those in the PDFs. Looking ahead, larger sensitivity and increased cross sections will be provided at the high-luminosity runs and high-energy upgrade of the LHC [17].

\section{References}

[1] M. Klasen, M. Pohl and G. Sigl, Prog. Part. Nucl. Phys. 85 (2015) 1.

[2] B. Fuks, M. Klasen, S. Schmiemann and M. Sunder, Eur. Phys. J. C 78 (2018) 209; M. Aaboud et al. [ATLAS Collaboration], Phys. Rev. D 98 (2018) 092002; A. M. Sirunyan et al. [CMS Collaboration], Phys. Lett. B 782 (2018) 440.

[3] W. Beenakker, M. Klasen, M. Krämer, T. Plehn, M. Spira and P. M. Zerwas, Phys. Rev. Lett. 83 (1999) 3780 Erratum: [Phys. Rev. Lett. 100 (2008) 029901].

[4] G. Bozzi, B. Fuks and M. Klasen, Phys. Rev. D 74 (2006) 015001; Nucl. Phys. B 777 (2007) 157; Nucl. Phys. B 794 (2008) 46; B. Fuks, M. Klasen, D. R. Lamprea and M. Rothering, JHEP 1401 (2014) 168 . 
[5] J. Debove, B. Fuks and M. Klasen, Phys. Lett. B 688 (2010) 208; Nucl. Phys. B 842 (2011) 51; Nucl. Phys. B 849 (2011) 64; B. Fuks, M. Klasen, D. R. Lamprea and M. Rothering, JHEP 1210 (2012) 081.

[6] W. Beenakker, R. Höpker, M. Spira and P. M. Zerwas, Nucl. Phys. B 492 (1997) 51; W. Beenakker, M. Krämer, T. Plehn, M. Spira and P. M. Zerwas, Nucl. Phys. B 515 (1998) 3.

[7] W. Beenakker, C. Borschensky, M. Krämer, A. Kulesza and E. Laenen, JHEP 1612 (2016) 133.

[8] M. Spira, hep-ph/0211145.

[9] B. Fuks, M. Klasen and M. Rothering, JHEP 1607 (2016) 053.

[10] L. M. Carpenter and K. Hendricks, arXiv:1812.08406 [hep-ph].

[11] B. Fuks, M. Klasen, D. R. Lamprea and M. Rothering, Eur. Phys. J. C 73 (2013) 2480.

[12] M. Bonvini et al., JHEP 1509 (2015) 191.

[13] W. Beenakker, C. Borschensky, M. Krämer, A. Kulesza, E. Laenen, S. Marzani and J. Rojo, Eur. Phys. J. C 76 (2016) 53.

[14] J. Fiaschi and M. Klasen, JHEP 1803 (2018) 094; Phys. Rev. D 98 (2018) 055014.

[15] G. Aad et al. [ATLAS Collaboration], arXiv:1908.08215 [hep-ex].

[16] A. M. Sirunyan et al. [CMS Collaboration], arXiv:1907.13179 [hep-ex].

[17] X. Cid Vidal et al. [Working Group 3], arXiv:1812.07831 [hep-ph]. 